\title{
ARANEOFAUNA (ARACHNIDA: ARANEAE) DEL ESTADO DE MÉXICO, MÉXICO
}

\author{
Marco Antonio DESALES-LARA \\ Centro de Investigación de Recursos Bióticos (CIRB), Universidad Autónoma del Estado de México, \\ Carretera Toluca-Ixtlahuaca Km 14.5 "Campus el Rosedal” San Cayetano de Morelos, Toluca, Estado \\ de México, México.<madesales@gmail.com>
}

Desales-Lara, M. A. 2014. Araneofauna (Arachnida: Araneae) del Estado de México, México. Acta Zoológica Mexicana (n. s.), 30(2): 298-320.

RESUMEN. Se presenta un listado de especies de arañas por municipio para el Estado de México, con base en una revisión bibliográfica. A la fecha se tienen registradas para la entidad 208 especies, agrupadas en 153 géneros y 42 familias. Las familias más diversas fueron Lycosidae, Araneidae, Theridiidae y Salticidae. Los municipios con mayor número de especies citadas fueron Ecatepec, Temascaltepec, Coacalco y Villa del Carbón.

Palabras clave: Araña, Diversidad, Araneae, Estado de México, Lista de especies.

Desales-Lara, M. A. 2014. Spider fauna (Arachnida: Araneae) from Estado de México, Mexico. Acta Zoológica Mexicana (n. s.), 30(2): 298-320.

ABSTRACT. A list of species of spider for each municipality from Estado de Mexico is given, based in a bibliographic revision. To date there are 208 species records, grouped in 153 genera and 42 families. The most diverse families were Lycosidae, Araneidae, Theridiidae and Salticidae. The municipalities with the most of the cited species were Ecatepec, Temascaltepec, Cocacalco and Villa del Carbon.

Key words: Spider, Diversity, Araneae, Estado de México, Species listing.

\section{INTRODUCCIÓN}

El orden Araneae ocupa el séptimo lugar en diversidad global (Coddington \& Levi 1991), con aproximadamente 44, 540 especies, agrupadas en 3, 924 géneros y 112 familias (Platnick 2014). No obstante, la araneofauna del mundo no esta completamente estudiada, los únicos lugares en donde la mayoría de las especies están mejor estudiadas es Japón, Oeste de Europa (especialmente Inglaterra) y Norteamérica (Jiménez 1996; Aguilera \& Casanueva 2005).

México cuenta con una araneofauna muy diversa, citándose hasta la fecha 2,295 especies (Francke 2011), 423 géneros y 64 familias (Aguayo-Morales et al. 2012). Pese a estos números, los trabajos son muy escasos y rezagados, adoleciendo de inventarios para el país y para cada uno de los estados que lo conforman. 
A la fecha sólo se cuentan con dos revisiones sobre la diversidad global de arañas en México: 1) la de Hoffman (1976), en donde se enlistan las especies de arañas presentes en cada estado de la República Mexicana, 2) la de Jiménez (1996), quien aporta una lista de las familias y géneros de arañas en México y el número aproximado de las especies por estado. Adicionalmente Durán-Barrón (2009), proporciona un listado de las arañas sinantrópicas de México y Platnick (2014), incluye citas a las publicaciones taxonómicas que han consignado las especies de arañas presentes en el país

Sin embargo, son casi muy pocos los trabajos en donde se documenten las especies de arañas presentes en cada una de las entidades de la República Mexicana. Chiapas es el único estado que cuenta con un listado especies de arañas publicado, con 464 especies, distribuidas en 281 géneros y 56 familias (García-Villafuerte 2009). Otros trabajos como el de Arenas-Monroy et al. (2012), quienes indican la presencia de 50 especies de arañas para el estado de Guanajuato y el de Lucio-Palacio (2012), que registra 19 especies [11 de su trabajo y 8 citas por Jiménez (1996)] para el estado de Aguascalientes.

Los registros de las especies de arañas para el Estado de México, se encuentran publicados en artículos, monografías y tesis sobre algunas familias o provienen de revisiones a nivel genérico con base en ejemplares depositados en colecciones nacionales o del extranjero. El primer trabajo donde se da un listado de especies de arañas para el estado, es el de Hoffman (1976), quien cita para la entidad 68 especies, posteriormente Platnick (1977), Platnick \& Shadad (1977, 1980, 1982), Gertsch \& Ennik (1983), Coyle (1988), Jiménez (1985a, 1985b, 1986, 1987), Jiménez- Jiménez (1989) y Jiménez \& Morales (1986), proporcionan más registros. En 1996, Jiménez cita para el Estado de México 126 especies de arañas, sin mencionar las especies presentes. Posteriormente, Durán-Barrón (2004), Olguín (2004), Levi (2005), Rheims et al. (2006), Platnick \& Ubick (2007), Desales-Lara et al. (2008), Valdez-Mondragón (2010), Estrada-Alvarez \& Locht (2011), Desales-Lara (2012), Desales-Lara et al. (2013), Guerrero-Fuentes \& Desales-Lara (2013) y Mendoza (2014), describen y citan especies para el estado. Pero sin duda la mayor cantidad de registros para la entidad lo proporcionan los trabajos de tesis de Doctorado de Jiménez-Jiménez (1989), la tesis de Maestría de Desales-Lara (2011), y las tesis de Licenciatura de Medina (2002), Desales-Lara (2009), Campuzano-Granados (2012), Rodríguez-Tapia (2013), Cruz (2014) y Guerrero-Fuentes (2014), ya que estos trabajos se han realizado dentro del Estado de México.

Debido a que la información sobre las especies de arañas para el Estado de México, se encuentra dispersa en un gran número de escritos y en ocasiones no se tiene fácil acceso a ello, el presente trabajo tiene como objetivo realizar un listado de las especies de arañas por municipio para el Estado de México. 


\section{MATERIAL Y MÉTODOS}

Para llevar a cabo este trabajo, se realizó una exhaustiva revisión bibliográfica de artículos científicos, en las bases de datos CAB Abstracts y Scopus; y se consultó el acervo bibliográfico de la Colección Nacional de Aracnidos (CNAN) del Instituto de Biología de la Universidad Nacional Autónoma de México (UNAM). De dichas consultas se obtuvieron los datos del municipio donde se recolectaron los organismos. Los nombres de las especies encontradas se corroboraron siguiendo la nomenclatura del World Spider Catalog (Platnick 2014).

En algunos trabajos consultados, se encontraron citas de organismos determinados solo a nivel familia y en otros casos solo a nivel de género pero carentes del epíteto específico, por tal motivo dentro del listado se encuentran algunas citas solo a nivel de familia o género.

Se elaboró un mapa del Estado de México en ArcGIS 10, utilizando la capa de división política estatal de México a una escala de 1:250000 y la capa municipios de la Comisión Nacional para el Conocimiento y Uso de la Biodiversidad (CONABIO 2012).

\section{RESULTADOS}

Se encontraron 34 trabajos en los cuales se citan especies de arañas para el Estado de México, de los cuales el 76\% son de aracnólogos mexicanos y el 24\% restante del extranjero. Con base en la información encontrada, el estado cuenta con 42 familias, 153 géneros, 208 especies de arañas, además de dos subespecies; lo que representa el $65.6 \%$ de las familias, el $36.1 \%$ de los géneros y $9 \%$ de la especies registradas para México, mientras que a nivel mundial los datos corresponden al $37.5 \%$ de las familias, el $3.8 \%$ de los géneros y apenas el $0.46 \%$ de las especies de la riqueza global. Del total de especies de arañas presentes en la entidad, 35 son sinantrópicas, de las cuales solo el $25.7 \%$ ( 9 especies) se registran tanto en el interior de las viviendas como en áreas naturales.

La familia con mayor número de especies en estado es: Lycosidae (34), seguido de Araneidae (24), Theridiidae (24), Salticidae (22), Gnaphosidae (15), Corinnidae (12) y Linyphiidae (12); mientras que el resto de las familias tienen 9 o menos especies.

La figura 1 muestra un mapa de los municipios del Estado de México en donde se tienen registros de especies de arañas, siendo Ecatepec el municipio con mayor número de especies citadas (22.3\%), seguido de Temascaltepec (17.1\%), Coacalco (16.6\%), Villa del Carbón (16.6\%), Tonatico (11.4\%) y Malinalco (10\%). Es importante indicar que del $25.7 \%$ de las especies, no se encontró el dato del municipio en el cual fueron recolectadas y sólo se citan para el Estado de México. 


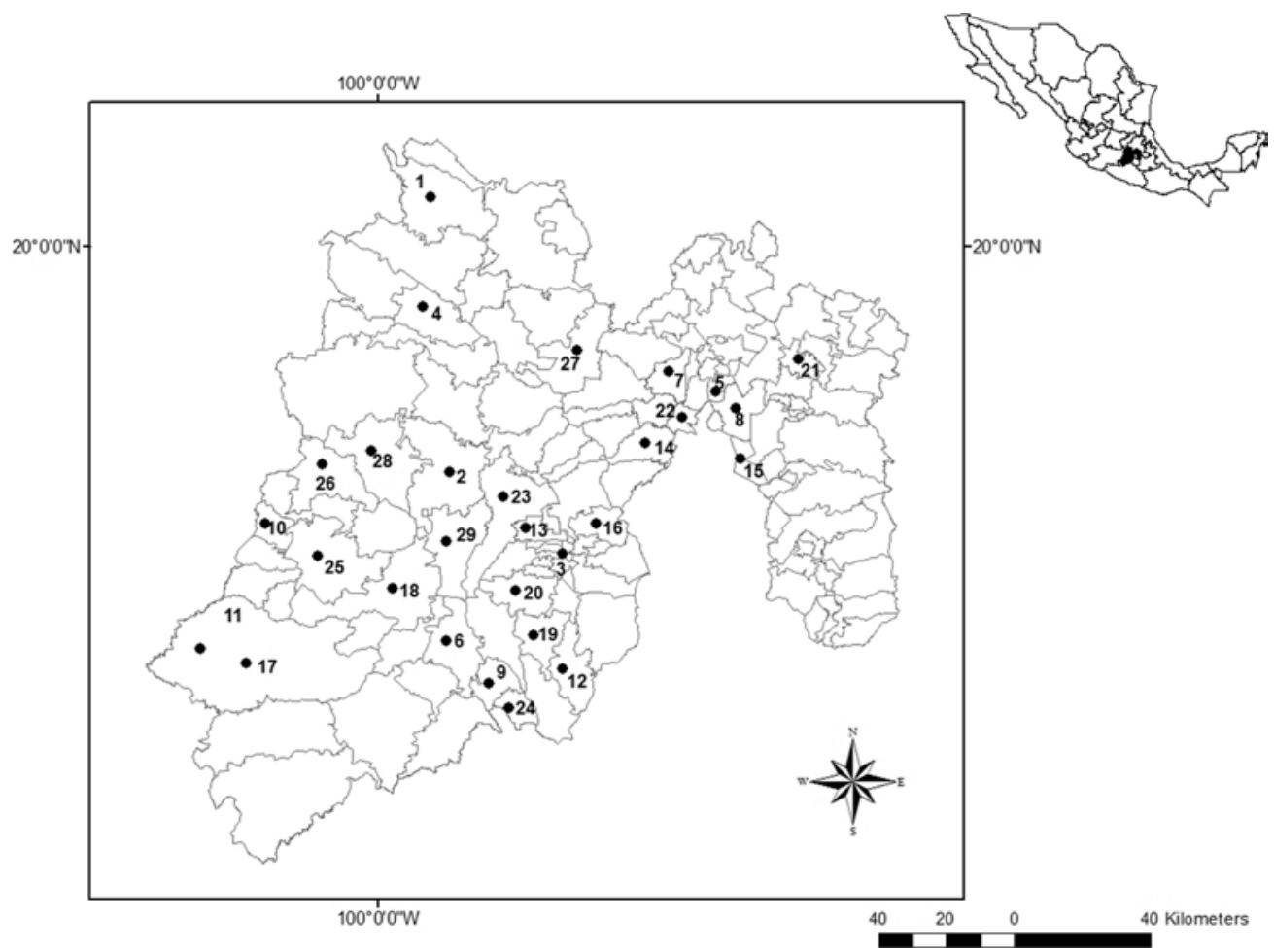

Figura 1. Mapa del Estado de México, en donde se indican con números los municipios que cuentan con registros de especies de arañas. El dígito entre el paréntesis después del nombre del municipio, indican el número de especies citadas para esa entidad. 1= Aculco (1). 2= Almoloya de Juárez (2). 3= Atizapán (11). 4= Atlacomulco (4). 5= Coacalco (35). 6= Coatepec Harinas (8). 7= Cuautitlán Izcalli (9). 8= Ecatepec (47). 9= Ixtapan de la Sal (1). 10= Ixtapan del Oro (1). 11= Luvianos (10). 12= Malinalco (21). 13= Metepec (6). 14= Naucalpan (17). 15= Nezahualcoyotl (4). 16= Ocoyoacac (1). $17=$ Tejupilco (1). 18= Temascaltepec (36). 19= Tenancingo (5). $20=$ Tenango del Valle (5). $21=$ Teotihuacán (3). 22= Tlalnepantla (12). 23= Toluca (10). 24= Tonatico (24). 25= Valle de Bravo (1). 26= Villa de Allende (1). 27= Villa del Carbón (35). 28= Villa Victoria (1). 29= Zinacantepec (1).

Se encontraron algunos cambios nomenclaturales (Cuadro 1) para algunas de las especies citadas en las fuentes consultadas con respecto a los nombres validos a la fecha (Platnick 2014), estos cambios ya están considerados en el listado de especies. 
Cuadro 1. Cambios nomenclaturales $(\mathrm{CN})$, de acuerdo con Platnick (2014). S= Sinonimia, $\mathrm{T}=$ Transferido, $\mathrm{ND}=$ Nomen dubium.

\begin{tabular}{|c|c|c|c|}
\hline Fuente (s) & Familia & Género y/o especie & $\mathrm{CN}$ \\
\hline Hoffmann (1976) & Agelenidae & Calymmaria modestella & ND \\
\hline Hoffmann (1976) & Anyphaenidae & Aysha cambridgei & T Hibana \\
\hline Hoffmann (1976) & Anyphaenidae & Aysha decepta & S Hibana futilis \\
\hline Hoffmann (1976) & Clubionidae & Castianeira plorans & T Corinnidae \\
\hline Hoffmann (1976) & Clubionidae & Mazax pax & $\mathrm{T}$ Corinnidae \\
\hline Hoffmann (1976) & Clubionidae & Trachelas deceptus & $\mathrm{T}$ Corinnidae \\
\hline $\begin{array}{l}\text { Hoffmann (1976) y Medina } \\
\text { (2002) }\end{array}$ & Corinnidae & Trachelas deceptus & T Meriola \\
\hline Hoffmann (1976) & Filistatidae & Filistata hibernalis & T Kukulcania \\
\hline Hoffmann (1976) & Gnaphosidae & Drassodes perditus & T Herpyllus \\
\hline Hoffmann (1976) & Gnaphosidae & Zelotes mexicanus & T Drassyllus \\
\hline Hoffmann (1976) & Linyphiidae & Frontinella pyramitela & S F. communis \\
\hline $\begin{array}{l}\text { Hoffmann (1976) y Medina } \\
\text { (2002) }\end{array}$ & Linyphiidae & Eperigone & S Mermessus \\
\hline Hoffmann (1976) & Lycosidae & Arctosa apora & $\mathrm{T}$ Allocosa \\
\hline Hoffmann (1976) & Salticidae & Phidippus fascipes & T Paraphidippus \\
\hline Hoffmann (1976) & Scytodiae & Scytodes mexicana & S S. univittata \\
\hline Hoffmann (1976) & Theraphosidae & Dugesiella & S Aphonopelma \\
\hline Hoffmann (1976) & Theraphosidae & Mygale mexicana & ND \\
\hline Jiménez-Jiménez (1989) & Agelenidae & Tortolena confusa & S T. glaucopis \\
\hline Jiménez-Jiménez (1989) & Clubionidae & Clubionoides & S Elaver \\
\hline Jiménez-Jiménez (1989) & Clubionidae & Phrurotimpus & $\mathrm{T}$ Corinnidae \\
\hline Jiménez-Jiménez (1989) & Clubionidae & Strotarchus & T Miturgidae \\
\hline Jiménez-Jiménez (1989) & Dictynidae & Dictyna oxtotilpanensis & T Emblyna \\
\hline Jiménez-Jiménez (1989) & Linyphiidae & $\begin{array}{l}\text { Selenyphantes } \\
\text { longispinosa }\end{array}$ & CE S. longispinosus \\
\hline Jiménez-Jiménez (1989) & Salticidae & Metaphiddipus furcatus & T Pelegrina \\
\hline $\begin{array}{l}\text { Jiménez-Jiménez (1989) } \\
\text { y Medina (2002) }\end{array}$ & Salticidae & Eris aurantia & T Paraphidippus \\
\hline Jiménez-Jiménez (1989) & Theridiidae & Episinus cognatus & T Neopisinus \\
\hline $\begin{array}{l}\text { Jiménez-Jiménez (1989) } \\
\text { y Durán-Barrón (2004) }\end{array}$ & Theridiidae & Achaearanea porteri & T Cryptachaea \\
\hline $\begin{array}{l}\text { Jiménez-Jiménez (1989) } \\
\text { y Durán-Barrón (2004) }\end{array}$ & Theridiidae & Episinus cognatus & T Neopisinus \\
\hline $\begin{array}{l}\text { Jiménez (1986) y Jiménez- } \\
\text { Jiménez (1989) }\end{array}$ & Thomisidae & Misumena vazquezae & T Misumenoides \\
\hline Medina (2002) & Corinnidae & Phrurolithus brittoni & T Scotinella \\
\hline Medina (2002) & Liocranidae & Phrurolithus brittoni & $\mathrm{T}$ Corinnidae \\
\hline
\end{tabular}


Cuadro 1. (Continúa).

\begin{tabular}{llll}
\hline Fuente (s) & Familia & Género y/o especie & CN \\
\hline Medina (2002) & Theridiidae & Dipoena furtiva & T Phycosoma \\
$\begin{array}{l}\text { Medina (2002) y Durán-Barrón } \\
\text { (2004) }\end{array}$ & Thomisidae & Misumenops decorus & T Mecaphesa \\
Olguín (2004) & Lycosidae & Hogna helluo & T Tigrosa \\
Desales-Lara (2009) & Cyrtaucheniidae & Eucteniza & T Euctenizidae \\
\hline
\end{tabular}

\section{LISTA DE ESPECIES DE ARAÑAS POR MUNICIPIO CITADAS PARA EL ESTADO DE MÉXICO, INCLUYENDO LA FUENTE DE DONDE PROVIENE LA INFORMACIÓN.}

Las familias aparecen en orden taxonómico (Platnick 2014), mientras que los géneros y las especies en orden alfabético. $(*=$ trabajos donde se cita a la especie como sinantrópica).

\section{Infraorden Mygalomorphae}

Familia Atypide. Tonatico (Guerrero-Fuentes \& Desales-Lara 2013).

Familia Dipluridae

Euagrus gus Coyle, 1988. Tenancingo, Tenango de Valle, Villa de Allende, Villa Victoria, Zinacantepec (Coyle 1988), Villa del Carbón (Medina 2002), Malinalco (Desales-Lara 2009), Coatepec Harinas (Campuzano-Granados 2012), Luvianos (Tapia-Rodríguez 2013), Coacalco, Ecatepec (Cruz 2014).

E. mexicanus Ausserer, 1875. Sólo se cita para el Estado de México (Hoffmann 1976).

Familia Ctenizidae

Hebestatis sp. Malinalco (Desales-Lara 2009).

Ummidia sp. Coatepec Harinas (Campuzano-Granados 2012).

Familia Euctenizidae

Eucteniza sp. Malinalco (Desales-Lara 2009).

Familia Theraphosidae

Aphonopelma seemanni (F. O. P.-Cambridge 1897). Sólo se cita para el Estado de México (Hoffmann 1976).

Aphonopelma sp. Malinalco (Desales-Lara 2009). Coacalco, Ecatepec (Cruz 2014).

Bonnetina aviae Estrada-Alvarez \& Locht 2011. Ecatepec (Estrada-Alvarez \& Locht 2011; Cruz 2014) Coacalco (Cruz 2014).

Hemirrhagus ocellatus Pérez-Miles \& Locht, 2003. Valle de Bravo (Mendoza 2014)

Metriopelma breyeri (Becker, 1878). Sólo se cita para el Estado de México (Hoffmann 1976). 


\section{Infraorden Araneomorphae}

Familia Filistatidae

Filistatinella domestica Desales-Lara, 2012. Almoloya de Juárez, Metepec, Toluca (Desales-Lara 2012)*.

Filistatoides sp. Atizapán, Ecatepec, Naucalpan (Durán-Barrón 2004)*.

Kukulcania hibernalis (Hentz, 1842). Atizapán, Naucalpan (Durán-Barrón 2004)*. Ecatepec (Durán-Barrón 2004*; Cruz 2014), Coacalco (Cruz 2014).

Kukulcania sp. Toluca (Desales-Lara 2011; Desales-Lara et al. 2013)*.

Familia Sicariidae

Loxosceles misteca Gertsch, 1958. Tonatico (Gertsch \& Ennik 1983).

Loxosceles sp. Malinalco (Desales-Lara 2009).

Familia Scytodidae

Scytodes intricata Banks, 1909. Tonatico (Rheims et al. 2006), Malinalco (Desales-Lara 2009).

S. longipes Lucas, 1844. Cuautitlan Izcalli, Naucalpan, Tlalnepantla (Durán-Barrón 2004)*, Toluca (Desales-Lara 2011; Desales-Lara et al. 2013)*, Coatepec Harinas (Campuzano-Granados 2012), Ecatepec (Cruz 2014).

S. univittata Simon, 1882. Sólo se cita para el Estado de México (Hoffmann 1976).

Familia Pholcidae

Crossopriza lyoni Blackwall, 1867. Coatepec Harinas (Campuzano-Granados 2012).

Pholcophora sp. Malinalco (Desales-Lara 2009).

Physocyclus dugesi Simon, 1893. Teotihuacán (Valdez-Mondragón 2010), Almoloya de Juárez, Metepec, Toluca (Desales-Lara 2011; Desales-Lara et al. 2013)*, Ecatepec (Valdez-Mondragón 2010; Cruz 2014).

P. globosus (Taczanowski, 1874). Atizapán, Cuautitlán Izcalli, Ecatepec, Naucalpan, Nezahualcoyotl, Tlalnepantla (Durán-Barrón 2004)*, Toluca (Desales-Lara 2011; Desales-Lara et al. 2013)*.

P. michoacanus Valdez-Mondragón, 2010. Tejupilco (Valdez-Mondragón 2010).

Psilochorus sp. Villa del Carbón (Medina 2002), Malinalco (Desales-Lara 2009), Almoloya de Juárez, Metepec, Toluca (Desales-Lara 2011; Desales-Lara et al. 2013)*, Coatepec Harinas (Campuzano-Granados 2012).

Familia Diguetidae

Diguetia propinqua (O. P.-Cambridge, 1896). Sólo se cita para el Estado de México (Hoffmann 1976).

Familia Dysderidae

Dysdera crocata C. L. Koch, 1838. Naucalpan (Durán-Barrón 2004)*, Ecatepec (Durán-Barrón 2004 (Durán-Barrón 2004*, Cruz 2014). 
Familia Oonopidae

Oonops sp. Almoloya de Juárez (Desales-Lara 2011; Desales-Lara et al. $2013)^{*}$.

Oonops tolucanus Gertsch \& Davis, 1942. Sólo se cita para el Estado de México (Hoffmann 1976).

Scaphiella sp. Malinalco (Desales-Lara 2009).

Familia Mimetidae

Mimetus hesperus Chamberlin, 1923. Atizapán (Durán-Barrón 2004).*

Familia Oecobiidae

Oecobius juangarcia Shear, 1970. Malinalco (Desales-Lara 2009).

O. navus Blackwall, 1859. Ecatepec (Durán-Barrón 2004).*

Familia Deinopidae

Deinopis sp. Malinalco (Desales-Lara 2009).

Familia Uloboridae

Hyptiotes sp. Villa del Carbón (Medina 2002).

Uloborus sp. Villa del Carbón (Medina 2002).

Familia Theridiidae

Anelosimus studiosus (Hentz, 1850). Atizapán, Naucalpan (Durán-Barrón 2004)*.

Argyrodes sp. Malinalco (Desales-Lara et al. 2008).

Cryptachaea porteri (Banks, 1896). Naucalpan (Durán-Barrón 2004)*.

C. pura (O. P.-Cambridge, 1894). Temascaltepec (Jiménez-Jiménez 1989).

Euryopis spinigera O. P.-Cambridge, 1895. Sólo se cita para el Estado de México (Hoffmann 1976).

Exalbidion pallisterorum (Levi, 1959). Temascaltepec (Jiménez-Jiménez 1989).

Faiditus subdolus (O. P.-Cambridge, 1898) Malinalco (Desales-Lara et al. 2008).

Latrodectus mactans (Fabricius, 1775). Cuautitlán Izcalli (Durán-Barrón 2004)*, Atlacomulco, Luvianos (Desales-Lara et al. 2008), Metepec (Desales-Lara 2011; Desales-Lara et al. 2013)*, Coacalco (Cruz 2014), Ecatepec (Durán-Barrón 2004*, Cruz 2014).

Neopisinus cognatus (O. P.-Cambridge, 1893). Temascaltepec (Jiménez-Jiménez 1989).

Nesticodes rufipes (Lucas, 1846). Sólo se cita para el Estado de México (Hoffmann 1976).

Phycosoma altum (Keyserling, 1886). Villa del Carbón (Medina 2002).

P. lineatipes (Bryant, 1933). Temascaltepec (Jiménez-Jiménez 1989).

Steatoda autumnalis (Banks, 1898). Coacalco, Ecatepec (Cruz 2014).

S. grossa (C. L. Koch, 1838). Atizapán, Cuautitlán Izcalli, Ecatepec, Naucalpan, Nezahualcoyotl, Tlalnepantla (Durán-Barrón 2004)*, Metepec (Desales-Lara et al. 
2008), Toluca (Desales-Lara 2011; Desales-Lara et al. 2013)*, Luvianos (Tapia-Rodríguez 2013).

S. saltensis Levi, 1957. Temascaltepec (Jiménez-Jiménez 1989).

S. variata china Gertsch, 1960. Solo se cita para el Estado de México (Hoffmann 1976).

Theridion adjacens (O. P.-Cambridge, 1896). Temascaltepec (Jiménez-Jiménez 1989), Villa del Carbón (Medina 2002).

T. australe Banks, 1899. Nezahualcoyotl (Durán-Barrón 2004).*

T. contreras Levi, 1959. Temascaltepec (Jiménez-Jiménez 1989), Villa del Carbón (Medina 2002).

T. coyoacan Levi, 1959. Naucalpan (Durán-Barrón 2004)*, Coacalco, Ecatepec (Cruz 2014).

T. crucum Levi, 1959. Temascaltepec (Jiménez-Jiménez 1989).

T. omiltemi Levi, 1959. Temascaltepec (Jiménez-Jiménez 1989).

T. rothi Levi, 1959. Temascaltepec (Jiménez-Jiménez 1989).

Theridion sp. Malinalco, Metepec (Desales-Lara et al. 2008), Almoloya de Juárez, Toluca (Desales-Lara 2011; Desales-Lara et al. 2013)*, Coacalco, Ecatepec (Cruz 2014).

T. styligerum F. O. P.-Cambridge, 1902. Temascaltepec (Jiménez-Jiménez 1989), Villa del Carbón (Medina 2002).

T. transgressum Petrunkevitch, 1911. Temascaltepec (Jiménez-Jiménez 1989).

Tidarren sisyphoides (Walckenaer, 1841). Malinalco (Desales-Lara et al. 2008). Familia Theridiosomatidae. Coatepec Harinas (Campuzano-Granados 2012).

Familia Linyphiidae

Ceratinopsis sp. Temascaltepec (Jiménez-Jiménez 1989).

Erigone dentosa O. P.-Cambridge, 1894. Solo se cita para el Estado de México (Hoffmann 1976).

E. tolucana Gertsch \& Davis, 1937. Solo se cita para el Estado de México (Hoffmann 1976).

Frontinella communis (Hentz, 1850). Solo se cita para el Estado de México (Hoffmann 1976).

F. huachuca Gertsch \& Davis, 1946. Villa del Carbón (Medina 2002).

F. huachuca benevola Gertsch \& Davis, 1946. Temascaltepec (Jiménez-Jiménez 1989).

Grammonota gentilis Banks, 1898. Sólo se cita para el Estado de México (Hoffmann 1976).

Jalaphyphantes minoratus Gertsch \& Davis, 1946. Solo se cita para el Estado de México (Hoffmann 1976).

Linyphia duplicata (F. O. P.-Cambridge, 1902). Solo se cita para el Estado de México (Hoffmann 1976). 
Meioneta sp. Temascaltepec (Jiménez-Jiménez 1989).

Mermessus agressus (Gertsch \& Davis, 1937). Villa del Carbón (Medina 2002).

M. annamae (Gertsch \& Davis, 1937). Sólo se cita para el Estado de México (Hoffmann 1976).

Mermessus sp. Se cita para el Estado de México (Hoffmann 1976).

Selenyphantes longispinosus (O. P.-Cambridge, 1896). Temascaltepec (JiménezJiménez 1989).

Walckenaeria crocea Millidge, 1983. Temascaltepec (Jiménez-Jiménez 1989).

Familia Tetragnathidae

Chrysometa brevipes (O. P.-Cambridge, 1889). Temascaltepec (Jiménez-Jiménez 1989).

C. puebla Levi, 1986. Villa del Carbón (Medina 2002).

Glenognatha sp. Temascaltepec (Jiménez-Jiménez 1989).

Leucauge mariana (Taczanowski, 1881). Naucalpan, Tlalnepantla (Durán-Barrón 2004)*.

Leucauge sp. Villa del Carbón (Medina 2002), Coacalco, Ecatepec (Cruz 2014).

Tetragnatha laboriosa Hentz, 1850. Temascaltepec (Jiménez-Jiménez 1989), Coacalco, Ecatepec (Cruz 2014).

T. versicolor Walckenaer, 1841. Temascaltepec (Jiménez-Jiménez 1989).

Familia Araneidae

Araneus guerrerensis Chamberlin \& Ivie, 1936. Temascaltepec (Jiménez-Jiménez 1989), Coacalco, Ecatepec (Cruz 2014).

A. rufipes O. P.-Cambridge, 1889. Ecatepec (Cruz 2014).

A. sinistrellus (Roewer, 1942). Solo se cita para el Estado de México (Hoffmann 1976).

A. tellezi Levi, 1991. Villa del Carbón (Medina 2002).

A. thaddeus (Hentz, 1847). Temascaltepec (Jiménez-Jiménez 1989), Atizapán (Durán-Barrón, 2004)*, Coacalco, Ecatepec (Cruz 2014).

Argiope argentata (Fabricius, 1775). Solo se cita para el Estado de México (Hoffmann 1976).

A. trifasciata (Forsskål, 1775). Coacalco (Cruz 2014).

Cyclosa caroli (Hentz, 1850). Temascaltepec (Jiménez-Jiménez 1989).

C. walckenaeri (O. P.-Cambridge, 1889). Temascaltepec (Jiménez-Jiménez 1989), Atlacomulco (Desales-Lara et al. 2008).

Cyclosa sp. Villa del Carbón (Medina 2002), Toluca (Desales-Lara 2011; Desales Lara et al. 2013)*.

Eriophora edax (Blackwall, 1863). Solo se cita para el Estado de México (Hoffmann 1976).

E. ravilla (C. L. Koch, 1844). Luvianos, Malinalco (Desales-Lara et al. 2008). 
Larinia famulatoria (Keyserling, 1883). Solo se cita para el Estado de México (Hoffmann 1976).

Mangora ixtapan Levi, 2005. Tenango del Valle (Levi 2005).

M. nahuatl Levi, 2005. Tenango del Valle (Levi 2005).

M. passiva (O. P.-Cambridge, 1889). Villa del Carbón (Medina 2002).

Metazygia sp. Luvianos (Desales-Lara et al. 2008).

Metepeira chilapae Chamberlin \& Ivie, 1936. Atlacomulco (Desales-Lara et al. 2008).

Metepeira sp. Villa del Carbón (Medina 2002), Tlalnepantla (Durán-Barrón 2004)*.

M. spinipes F. O. P.-Cambridge, 1903. Coacalco, Ecatepec (Cruz 2014).

Micrathena gracilis (Walckenaer, 1805). Malinalco (Desales-Lara et al. 2008).

M. mitrata (Hentz, 1850). Malinalco (Desales-Lara et al. 2008).

Neoscona arabesca (Walckenaer, 1841). Solo se cita para el Estado de México (Hoffmann 1976).

N. nautica (L. Koch, 1875). Solo se cita para el Estado de México (Hoffmann 1976).

N. oaxacensis (Keyserling, 1864). Tlalnepantla (Durán-Barrón 2004)*, Malinalco, Metepec (Desales-Lara et al. 2008), Coacalco, Ecatepec (Cruz 2014).

N. orizabensis F. O. P.-Cambridge, 1904. Temascaltepec (Jiménez-Jiménez 1989), Villa del Carbón (Medina, 2002), Metepec (Desales-Lara et al. 2008), Coatepec Harinas (Campuzano-Granados 2012), Coacalco, Ecatepec (Cruz 2014).

Verrucosa arenata (Walckenaer, 1841). Malinalco (Desales-Lara et al. 2008).

Familia Lycosidae

Allocosa apora (Gertsch, 1934). Temascaltepec (Jiménez-Jiménez 1989).

Allocosa sp. Tlalnepantla (Durán-Barrón 2004)*.

A. veracruzana (Gertsch \& Davis, 1940). Naucalpan (Durán-Barrón 2004*; Olguín 2004), Malinalco (Desales-Lara 2009).

Alopecosa sp. Malinalco (Desales-Lara 2009), Tonatico (Guerrero-Fuentes 2014).

Arctosa denticulata Jiménez \& Dondale, 1984. Se cita para el Estado de México (Olguín 2004).

A. minuta F. O. P.-Cambridge, 1902. Naulcalpan (Durán-Barrón 2004*; Olguín 2004).

Arctosa sp. Toluca (Desales-Lara 2011; Desales-Lara et al. 2013)*.

Hogna lenta (Hentz, 1844). Solo se cita para el Estado de México (Olguín 2004).

Hogna sp. Tonatico (Guerrero-Fuentes 2014).

Pardosa altamontis Chamberlin \& Ivie, 1946. Solo se cita para el Estado de México (Olguín 2004). 
P. amacuzacensis Jiménez, 1983. Solo se cita para el Estado de México (Olguín 2004).

P. dondalei Jiménez, 1986. Temascaltepec (Jiménez 1986a; Jiménez-Jiménez 1989).

P. ecatli Jiménez, 1985. Valle de Bravo (Jiménez 1985; Olguín 2004).

P. falcifera F. O. P.-Cambridge, 1902. Atizapán, Cuautitlán Izcalli (Durán-Barrón 2004)*, Coacalco, Ecatepec (Cruz 2014).

P. flavipalpis F. O. P.-Cambridge, 1902. Solo se cita para el Estado de México (Olguín 2004).

P. guadalajarana Dondale \& Redner, 1984. Solo se cita para el Estado de México (Olguín 2004).

P. marialuisae Dondale \& Redner, 1984. Tonatico (Guerrero-Fuentes 2014).

P. medialis Banks, 1898. Tonatico (Guerrero-Fuentes 2014).

P. montgomeryi Gertsch, 1934. Solo se cita para el Estado de México (Olguín 2004).

P. pauxilla Montgomery, 1904. Tonatico (Guerrero-Fuentes 2014).

P. petrunkevitchi Gertsch, 1934. Temascaltepec (Jiménez-Jiménez 1989), Villa del Carbón (Medina 2002).

P. prolifica F. O. P.-Cambridge, 1902. Solo se cita para el Estado de México (Olguín 2004).

Pardosa sp. Tlalnepantla (Durán-Barrón 2004)*, Malinalco (Desales-Lara 2009), Toluca (Desales-Lara 2011; Desales-Lara et al. 2013)*, Coatepec Harinas (Campuzano-Granados 2012), Luvianos (Tapia-Rodríguez 2013), Coacalco, Ecatepec (Cruz 2014), Tonatico (Guerrero-Fuentes 2014).

P. sternalis (Thorell, 1877). Villa del Carbón (Medina 2002; Olguín 2004).

P. steva Lowrie \& Gertsch, 1955. Solo se cita para el Estado de México (Olguín 2004).

P. tumida Barnes, 1959. Solo se cita para el Estado de México (Hoffmann 1976).

P. valens Barnes, 1959. Coacalco, Ecatepec (Cruz 2014).

P. vancouveri Emerton, 1917. Solo se cita para el Estado de México (Olguín 2004).

P. xerophila Vogel, 1964. Solo se cita para el Estado de México (Olguín 2004).

Pirata piraticus (Clerck, 1757). Solo se cita para el Estado de México (Olguín 2004).

Rabidosa rabida (Walckenaer, 1837). Solo se cita para el Estado de México (Olguín 2004).

R. santrita (Chamberlin \& Ivie, 1942). Ecatepec (Durán-Barrón 2004*; Olguín 2004; Cruz 2014). Coacalco (Cruz 2014).

Rabidosa sp. Cuautitlán Izcalli (Durán-Barrón 2004)*. 
Schizocosa avida (Walckenaer, 1837). Cuautitlán Izcalli, Ecatepec (Durán-Barrón 2004)*.

S. mccooki (Montgomery, 1904). Solo se cita para el Estado de México (Olguín 2004).

S. mimula (Gertsch, 1934). Solo se cita para el Estado de México (Olguín 2004).

S. minnesotensis (Gertsch, 1934). Solo se cita para el Estado de México (Olguín 2004).

Schizocosa sp. Villa del Carbón (Medina 2002).

Sosippus michoacanus Brady, 1962. Solo se cita para el Estado de México (O1guín 2004).

S. plutonus Brady, 1962. Solo se cita para el Estado de México (Hoffmann 1976).

Tigrosa helluo (Walckenaer, 1837)*. Solo se cita para el Estado de México (Olguín 2004).

Varacosa sp. Luvianos (Tapia-Rodríguez 2013).

Familia Pisauridae

Dolomedes triton (Walckenaer, 1837). Solo se cita para el Estado de México (Hoffmann 1976).

Familia Oxyopidae

Oxyopes sp. Malinalco (Desales-Lara 2009), Coacalco (Cruz 2014), Tonatico (Guerrero-Fuentes 2014).

Peucetia longipalpis F. O. P.-Cambridge, 1902. Solo se cita para el Estado de México (Hoffmann 1976).

Peucetia sp. Villa del Carbón (Medina 2002).

$P$. viridians (Hentz, 1832). Coacalco, Ecatepec (Cruz 2014).

Familia Zorocratidae

Zorocrates contreras Platnick \& Ubick, 2007. Villa del Carbón (Platnick \& Ubick, 2007).

Z. fuscus Simon, 1888. Atizapán, Cuautitlán Izcalli, Naucalpan, Tlalnepantla (Durán-Barrón 2004)*, Ecatepec (Durán-Barrón 2004*; Cruz 2014), Aculco, Malinalco, Naucalpan, Teotihuacán, Tenancingo, Tenango del Valle, Tonatico (Platnick \& Ubick 2007), Malinalco (Desales-Lara 2009), Toluca (Desales-Lara 2011; DesalesLara et al. 2013)*, Coatepec Harinas (Campuzano-Granados 2012), Luvianos (TapiaRodríguez 2013), Coacalco (Cruz 2014), Tonatico (Guerrero-Fuentes 2014).

Z. unicolor (Banks, 1901). Atlacomulco, Malinalco, Tenancingo (Platnick \& Ubick, 2007).

Familia Ctenidae

Ctenus sp. Solo se cita para el Estado de México (Hoffmann 1976).

Leptoctenus sp. Malinalco (Desales-Lara 2009), Coatepec Harinas (Campuzano-Granados 2012), Luvianos (Tapia-Rodríguez 2013), Tonatico (Guerrero-Fuentes 2014). 
Familia Agelenidae

Agelenopsis aperta (Gertsch, 1934) Ecatepec (Cruz 2014).

Agelenopsis sp. Coatepec Harinas (Campuzano-Granados 2012).

Barronopsis Chamberlin \& Ivie, 1941. Naucalpan (Durán-Barrón 2004)*.

Melpomene elegans O. P.-Cambridge, 1898 *. Tonatico (Guerrero-Fuentes 2014).

Novalena annamae (Gertsch \& Davis, 1940). Villa del Carbón (Medina 2002).

N. approximata (Gertsch \& Ivie, 1936). Naucalpan, Tlalnepantla (Durán-Barrón 2004)*.

Novalena sp. Coacalco, Ecatepec (Cruz 20014).

N. tolucana (Gertsch \& Davis, 1940). Villa del Carbón (Medina 2002).

Tegenaria domestica (Clerck, 1757). Atizapán, Ecatepec, Naulcalpan, Nezahualcoyotl, Tlalnepantla (Durán-Barrón 2004)*.

T. flexuosa F. O. P.-Cambridge, 1902. Solo se cita para el Estado de México (Hoffmann 1976).

T. mexicana Roth, 1968. Villa del Carbón (Medina 2002).

Tegenaria sp. Almoloya de Juárez, Metepec, Toluca (Desales-Lara, 2011; Desales-Lara et al. 2013)*.

Tortolena glaucopis (F. O. P.-Cambridge, 1902). Temascaltepec (Jiménez-Jiménez 1989), Villa del Carbón (Medina 2002), Coacalco, Ecatepec (Cruz 20014)

Tortolena sp. Malinalco (Desales-Lara 2009).

Familia Cybaeidae. Malinalco (Desales-Lara 2009), Coatepec Harinas (CampuzanoGranados 2012), Luvianos (Tapia-Rodríguez 2013).

Familia Hahniidae

Antistea sp. Coatepec Harinas (Campuzano-Granados 2012), Luvianos (TapiaRodríguez 2013).

Familia Dictynidae

Cicurina arcuata Keyserling, 1887. Villa del Carbón (Medina 2002).

Emblyna oxtotilpanensis (Jiménez \& Luz, 1986). Temascaltepec (Jiménez \& Morales 1986; Jiménez-Jiménez 1989).

Mallos gertschi Bond \& Opell, 1997. Villa del Carbón (Medina 2002).

Mallos sp. Atizapán, Cuautitlán Izcalli (Durán-Barrón 2004)*.

Mexitlia sp. Malinalco (Desales-Lara 2009).

Familia Tengellidae

Lauricius hemicloeinus Simon, 1888*. Coacalco (Cruz 2014).

Familia Miturgidae

Cheiracanthium inclusum (Hentz, 1847). Atizapán, Tlalnepantla (Durán-Barrón 2004)*, Coacalco, Ecatepec (Cruz 2014).

Strotarchus sp. Naucalpan (Durán-Barrón 2004)*, Villa del Carbón (Medina 2002). 
Familia Anyphaenidae

Anyphaena catalina Platnick, 1974. Villa del Carbón (Medina 2002), Tonatico (Guerrero-Fuentes 2014)

A. cortes Platnick \& Lau, 1975. Ocoyoacac (Platnick \& Lau 1975), Villa del Carbón (Medina 2002).

A. cumbre Platnick \& Lau, 1975. Toluca (Platnick \& Lau 1975).

A. judicata O. P.-Cambridge, 1896. Ixtapan de la Sal, Tenancingo, Tenango del Valle, Toluca (Platnick \& Lau 1975).

A. obregon Platnick \& Lau, 1975. Atizapán, Naucalpan (Durán-Barrón, 2004)*. Anyphaena sp. Villa del Carbón (Medina 2002), Malinalco (Desales-Lara, 2009), Toluca (Desales-Lara 2011; Desales-Lara et al. 2013)*, Coatepec Harinas (Campuzano-Granados 2012), Coacalco, Ecatepec (Cruz 2014).

A. xochimilco Platnick \& Lau, 1975. Ecatepec (Cruz 2014).

Hibana cambridgei (Bryant, 1931). Solo se cita para el Estado de México (Hoffmann 1976).

H. futilis (Banks, 1898). Tlalnepantla (Durán-Barrón 2004)*.

Familia Clubionidae

Clubiona sp. Ecatepec (Cruz 2014).

Elaver sp. Temascaltepec (Jiménez-Jiménez 1989), Tonatico (Guerrero-Fuentes 2014).

Familia Corinnidae

Castianeira azteca Reiskind, 1969. Tonatico (Guerrero-Fuentes 2014).

Castianeira luctifera Petrunkevitch, 1911. Villa del Carbón (Medina 2002).

C. plorans (O. P.-Cambridge, 1898). Sólo se cita para el Estado de México (Hoffmann 1976).

Castianeira sp. Ecatepec (Cruz 2014), Tonatico (Guerrero-Fuentes 2014).

C. stylifera Kraus, 1955. Malinalco (Desales-Lara 2009), Tonatico (GuerreroFuentes 2014).

C. truncata Kraus, 1955. Villa del Carbón (Medina 2002), Malinalco (DesalesLara 2009), Coatepec Harinas (Campuzano-Granados 2012), Luvianos (Tapia-Rodríguez 2013).

Creugas bicuspis (F. O. P.-Cambridge, 1899). Luvianos (Tapia-Rodríguez 2013).

C. gulosus Thorell, 1878. Malinalco (Desales-Lara 2009), Luvianos (Tapia-Rodríguez 2013), Tonatico (Guerrero-Fuentes 2014).

Creugas sp. Tonatico (Guerrero-Fuentes 2014).

Mazax pax Reiskind, 1969. Solo se cita para el Estado de México (Hoffmann 1976).

Meriola decepta Banks, 1895. Villa del Carbón (Medina 2002), Tonatico (Guerrero-Fuentes 2014). 
Phrurotimpus sp. Temascaltepec (Jiménez-Jiménez 1989).

Scotinella brittoni (Gertsch, 1941). Villa del Carbón (Medina 2002).

Trachelas cf. bulbosus F. O. P.-Cambridge, 1899. Toluca (Desales-Lara 2011)*.

T. depressus Platnick \& Shadab, 1974. Tonatico (Guerrero-Fuentes 2014).

Trachelas sp. Malinalco (Desales-Lara 2009), Coatepec Harinas (CampuzanoGranados 2012).

Familia Prodidomidae. Malinalco (Desales-Lara 2009).

Familia Gnaphosidae

Cesonia classica Chamberlin, 1924. Villa del Carbón (Medina 2002).

C. cuernavaca Platnick \& Shadab, 1980. Malinalco (Desales-Lara 2009), Coatepec Harinas (Campuzano-Granados 2012), Luvianos (Tapia-Rodríguez 2013), Tonatico (Guerrero-Fuentes 2014).

C. lugubris (O. P.-Cambridge, 1896). Toluca (Platnick \& Shadab, 1980) Malinalco (Desales-Lara 2009), Luvianos (Tapia-Rodríguez 2013), Tonatico (GuerreroFuentes 2014).

Drassodes sp. Luvianos (Tapia-Rodríguez 2013), Ecatepec (Cruz 2014).

Drassyllus coajus Platnick \& Shadab, 1982. Ixtapan del Oro (Platnick \& Shadab, 1982).

D. lepidus (Banks, 1899). Teotihuacán (Platnick \& Shadab, 1982).

D. mexicanus (Banks, 1898). Solo se cita para el Estado de México (Hoffmann 1976).

Drassyllus sp. Coatepec Harinas (Campuzano-Granados 2012), Coacalco, Ecatepec (Cruz 2014), Tonatico (Guerrero-Fuentes 2014).

Gnaphosa hirsutipes Banks, 1901. Tenancingo (Platnick \& Shadab, 1975), Villa del Carbón (Medina 2002).

Herpyllus brachet Platnick \& Shadab, 1977. Ecatepec (Durán-Barrón 2004)*, Tonatico (Guerrero-Fuentes 2014).

H. perditus (Banks, 1898). Coacalco, Ecatepec (Cruz 2014).

H. perote Platnick \& Shadab, 1977. Villa del Carbón (Medina 2002).

H. cf. reservatus Chamberlin, 1936. Malinalco (Desales-Lara 2009).

Herpyllus sp. Almoloya de Juárez, Metepec, Toluca (Desales-Lara 2011; DesalesLara et al. 2013)*, Coatepec Harinas (Campuzano-Granados 2012).

Nodocion floridanus (Banks, 1896). Cuautitlán Izcalli (Durán-Barrón 2004)*.

Trachyzelotes sp. Malinalco (Desales-Lara 2009).

Urozelotes rusticus (L. Koch, 1872). Ecatepec (Durán-Barrón 2004)*.

Zelotes anglo Gertsch \& Riechert, 1976. Coacalco, Ecatepec (Cruz 2014).

Zelotes moestus (O. P.-Cambridge, 1898). Villa del Carbón (Medina 2002), Coacalco, Ecatepec (Cruz 2014).

Zelotes sp. Luvianos (Tapia-Rodríguez 2013). 
Familia Selenopidae

Selenops sp. Malinalco (Desales-Lara 2009), Tonatico (Guerrero-Fuentes 2014). Familia Sparassidae

Heteropoda sp. Toluca (Desales-Lara 2011; Desales-Lara et al. 2013)*.

Olios sp. 1837. Malinalco (Desales-Lara 2009), Tonatico (Guerrero-Fuentes 2014).

Familia Philodromidae

Apollophanes longipes (O. P.-Cambridge, 1896). Temascaltepec (Jiménez-Jiménez 1989).

Apollophanes sp. Coacalco, Ecatepec (Cruz 2014).

A. texanus Banks, 1904. Villa del Carbón (Medina 2002).

Ebo sp. Ecatepec (Cruz 2014).

Philodromus sp. Temascaltepec (Jiménez-Jiménez 1989), Ecatepec (Cruz 2014).

Tibellus oblongus (Walckenaer, 1802)*. Ecatepec (Cruz 2014).

Familia Thomisidae

Bassaniana versicolor (Keyserling, 1880)*. Coacalco (Cruz 2014).

Mecaphesa celer (Hentz, 1847). Coacalco, Ecatepec (Cruz 2014).

M. decora (Banks, 1898). Villa del Carbón (Medina 2002), Atizapán (Durán-Barrón 2004)*.

Misumena sp. Tlalnepantla (Durán-Barrón 2004)*.

Misumenoides annulipes (O. P.-Cambridge 1891). Villa del Carbón (Medina 2002).

Misumenoides sp. Coacalco, Ecatepec (Cruz 2014).

M. vazquezae Jiménez, 1986. Temascaltepec (Jiménez 1986b; Jiménez-Jiménez 1989).

Misumenops sp. Temascaltepec (Jiménez-Jiménez 1989), Metepec, Toluca (Desales-Lara 2011; Desales-Lara et al. 2013)*.

Synema lopezi Jiménez, 1988. Temascaltepec (Jiménez 1987; Jiménez-Jiménez 1989).

Tmarus sp. Villa del Carbón (Medina 2002), Coacalco (Cruz 2014).

Tmarus tamazolinus Jiménez, 1988. Temascaltepec (Jiménez 1987; Jiménez-Jiménez 1989).

Xysticus paiutus Gertsch, 1933. Cuautitlán Izcalli, Tlalnepantla (Durán-Barrón 2004)*, Tonatico (Guerrero-Fuentes 2014).

X. pellax O.P. Cambridge, 1894. Coatepec Harinas (Campuzano-Granados 2012), Tonatico (Guerrero-Fuentes 2014).

Xysticus sp. Malinalco (Desales-Lara 2009), Ecatepec (Cruz 2014).

Familia Salticidae

Anicius sp. Temascaltepec (Jiménez-Jiménez 1989).

Chalcoscirtus sp. Coatepec Harinas (Campuzano-Granados 2012). 
Corythalia nigriventer (F. O. P.-Cambridge, 1901). Temascaltepec (Jiménez-Jiménez 1989).

Eris sp. Atizapán, Naucalpan, Tlalnepantla (Durán-Barrón 2004)*.

Habronattus altanus (Gertsch, 1934). Temascaltepec (Jiménez-Jiménez 1989).

H. anepsius (Chamberlin, 1924). Tonatico (Guerrero-Fuentes 2014).

H. fallax (Peckham \& Peckham, 1909). Malinalco (Desales-Lara 2009), Coacalco, Ecatepec (Cruz 2014), Tonatico (Guerrero-Fuentes 2014).

H. mexicanus (Peckham \& Peckham 1896). Tlalnepantla (Durán-Barrón 2004)*, Tonatico (Guerrero-Fuentes 2014).

H. pugillis Griswold, 1987. Tonatico (Guerrero-Fuentes 2014).

Habronattus sp. Luvianos (Tapia-Rodríguez 2013).

Marpissa sp. Tonatico (Guerrero-Fuentes 2014).

Metacyrba sp. Malinalco (Desales-Lara 2009).

M. taeniola (Hentz, 1846) *. Tonatico (Guerrero-Fuentes 2014).

Metaphidippus sp. Temascaltepec (Jiménez-Jiménez 1989).

Mexigonus minutus (F. O. P.-Cambridge, 1901). Malinalco (Desales-Lara 2009), Coatepec Harinas (Campuzano-Granados 2012), Coacalco, Ecatepec (Cruz 2014), Tonatico (Guerrero-Fuentes 2014).

Mexigonus sp. Atizapán (Durán-Barrón, 2004)*, Almoloya de Juárez, Metepec, Toluca (Desales-Lara 2011; Desales-Lara et al. 2013)*. Luvianos (Tapia-Rodríguez 2013), Coacalco, Ecatepec (Cruz 2014), Tonatico (Guerrero-Fuentes 2014).

Naphrys sp. Malinalco (Desales-Lara 2009).

Paramarpissa piratica (Peckham \& Peckham, 1888). Ecatepec (Cruz 2014).

Paraphidippus aurantius (Lucas, 1833). Temascaltepec (Jiménez-Jiménez 1989), Villa del Carbón (Medina 2002), Coacalco, Ecatepec (Cruz 2014).

P. fuscipes (C. L. Koch, 1846). Solo se cita para el Estado de México (Hoffmann 1976).

Peckhamia sp. Ecatepec (Cruz 2014).

Pelegrina bunites Maddison, 1996. Villa del Carbón (Medina 2002).

P. edrilana Maddison, 1996. Coacalco, Ecatepec (Cruz 2014).

P. furcata (F. O. P.-Cambridge, 1901). Temascaltepec (Jiménez-Jiménez 1989).

P. morelos Maddison, 1996. Villa del Carbón (Medina 2002).

P. variegata.(F. O. P.-Cambridge, 1901). Coacalco, Ecatepec (Cruz 2014).

Phanias sp. Villa del Carbón (Medina 2002).

Phidippus carneus Peckham \& Peckham, 1896. Coacalco, Ecatepec (Cruz 2014).

P. cerberus Edwards, 2004. Coacalco, Ecatepec (Cruz 2014).

P. octopunctatus (Peckham \& Peckham, 1883). Coacalco, Ecatepec (Cruz 2014).

Phidippus sp. Coacalco, Ecatepec (Cruz 2014).

Plexippus paykulli (Audouin, 1826). Naucalpan (Durán-Barrón 2004)*. 
P. seladonicus C. L. Koch, 1846. Solo se cita para el Estado de México (Hoffmann 1976).

Sarinda sp. Tonatico (Guerrero-Fuentes 2014).

Sassacus alboguttatus (F. O. P.-Cambridge, 1901). Coacalco, Ecatepec (Cruz 2014).

Sitticus sp. Tonatico (Guerrero-Fuentes 2014).

Thiodina sp. Tonatico (Guerrero-Fuentes 2014).

Tylogonus sp. Villa del Carbón (Medina 2002).

Zygoballus sp. 1885. Temascaltepec (Jiménez-Jiménez 1989).

\section{DISCUSIÓN}

Algunas de las especies incluidas en la lista precedente no aparecen citadas para México en el Word Spider Catalog (Platnick, 2014), ya que son trabajos de tesis que no han sido publicados y por lo tanto la información se conoce sólo localmente: Jiménez-Jiménez (1989) (Corythalia nigriventer de la familia Salticidae); Medina (2002) (Phycosoma altum de la familia Theridiidae; Frontinella huachuca de la familia Linyphiidae; Cicurina arcuata de la familia Dictynidae; Castianeira luctifera, C. truncata y Scotinella brittoni de la familia Corinnidae); Olguín (2004) (Tigrosa helluo, H. lenta, Pardosa altamontis, P. vancouveri, Rabidosa santrita y Schizocosa minnesotensis de la familia Lycosidae); Durán-Barrón (2004) (Mimetus hesperus de la familia Mimetidae; Novalena approximata de la familia Agelenidae), Desales-Lara (2009) (Castiaeneira stylifera de la familia Corinnidae), Campuzano-Granados (2012) (Castianeira truncata de la familia Corinnidae), Cruz (2014) (Araneus rufipes de la familia Araneidae) y Guerrero-Fuentes (2014) (Pardosa pauxilla de la familia Lycosidae).

Asimismo los géneros: Tylogonus de la familia Salticidae (Medina 2002); Barronopsis de la familia Agelenidae (Durán-Barrón 2004), Hebestatis de la familia Ctenizidae (Desales-Lara 2009), Antistea de la familia Hahniidae (Campuzano-Granados 2012; Tapia-Rodríguez 2013), Chalcoscirtus de la familia Salticidae (CampuzanoGranados 2012) y Sarinda (Guerrero-Fuentes 2014) no tienen registros en México (Platnick, 2014); lo mismo ocurre con la familia Cybaeidae, sin embargo ya es citada para el país por Desales-Lara (2009), Ibarra-Núñez et al. (2011), Campuzano-Granados (2012) y Tapia-Rodríguez (2013).

Medina (2002) y Durán-Barrón (2004) citan Zorocrates guerrerensis, para el Estado de México; sin embargo en la revisión del género Zorocrates por Platnick \& Ubick (2007), examinaron el material determinado por Medina (2002) y DuránBarrón (2004), encontrando que corresponde a la especies $Z$. contreras y $Z$. fuscus respectivamente, por lo tanto la especie $Z$. guerrerensis no se encuentra presente en el Estado de México. 
Pardosa sierra, es citada para el Estado de México por Hoffmann (1976), sin embargo Correa-Ramírez et al. (2010), la validan únicamente para la Península de Baja California, llegándola a considerar como endémica de esa zona.

Jiménez (1996), sitúa al Estado de México en 13avo lugar de diversidad de arañas en la República Mexicana, con el presente trabajo el estado se ubica en el 8avo, por el incremento de especies de 126 a 208. Esto indica que el conocimiento de la araneofauna de la entidad se ha incrementado en un $65 \%$; además es importante mencionar que si tomamos en cuenta las morfoespecies citadas en los trabajos de Jiménez (1989), Medina (2002), Durán-Barrón (2004), Desales-Lara et al. (2008), DesalesLara (2009), Desales-Lara (2011), Campuzano-Granados (2012), Desales-Lara et al. (2013), Tapia-Rodríguez (2013), Cruz (2014) y Guerrero-Fuentes (2014), el número de especies ascendería a 292 aproximadamente, lo que ubicaría al Estado de México en el cuarto lugar de diversidad de arañas, sólo por debajo de los estados de Chiapas, Baja California y Veracruz, (Jiménez 1996; García-Villafuerte 2009); sin embargo este aumento se lograra hasta que se determinen a los organismos a nivel de especie y los resultados sean publicados.

Ecatepec el municipio con mayor número de especies citadas $(22.3 \%)$, seguido de Temascaltepec (17.1\%), Coacalco (16.6\%), Villa del Carbón (16.6\%), Tonatico (11.4\%) y Malinalco (10\%). Es importante indicar que del $25.7 \%$ de las especies, no se encontró el dato del municipio en el cual fueron recolectadas y sólo se citan para el Estado de México.

En algunos municipios del estado se han realizado estudios anuales de arañas, lo que se ve reflejado en que el mayor número de especies registradas es para esas zonas (Fig. 1), como el trabajo de Cruz (2014), realizado en Ecatepec (municipio con mayor número de especies citadas) y Coacalco (municipio con el tercer lugar de especies registradas), el de Jiménez (1989) y el de Medina (2002) realizados en Temascaltepec y Villa del Carbón respectivamente (municipios con el segundo y cuarto lugar con mayor número de especies registradas).

De los 125 municipios que integran el Estado de México, sólo se tienen registros de al menos una especie de araña en 29 de ellos (23.2\%), este déficit indica que es necesario continuar con los estudios faunísticos en los municipios con menor número de especies registradas. También es necesario hacer un mayor esfuerzo para incrementar las revisiones taxonómicas de la fauna de este estado como de la fauna mexicana en general.

AGRADECIMIENTOS. Al Dr. Gabriel A. Villegas-Guzmán y a la M. en C. Griselda Montiel-Parra por la revisión y comentarios al manuscrito, así como a la CNAN por la literatura proporcionada. Al Biól. Giovany Arturo González-Desales por la ayuda en la elaboración del mapa de distribución. Al Dr. Alejandro Valdez-Mondragón. Además de los tres revisores anónimos por sus atinados comentarios para mejorar el escrito. Dedicado al M. en C. Jorge Padilla Ramírez y a los Dres. Cesar Gabriel Durán- 
Barrón y Oscar F. Francke B., quienes contribuyeron en mi gusto por los insectos, las arañas y el resto de los arácnidos respectivamente, además de participar en mi preparación profesional.

\section{LITERATURA CITADA}

Aguayo-Morales, C., Castelo-Calvillo, J. \& Víctor-Rosas, L. 2012. Análisis de la diversidad y endemismo de arácnidos (Excl. Acari) (Arthropoda:Chelicerata) de México, pp. 94-99. In: E. Estrada, A. Equihua, J. Acuña, M. Chaires \& G. Durán (Eds.). Entomología Mexicana Vol. 10. Sociedad Mexicana de Entomología, México.

Aguilera, M. A. \& Casanueva. M. E. 2005. Arañas Chilenas: estado actual del conocimiento y clave para las familias de Araneomorphae. Gayana, 69: 201-224.

Arenas-Monroy, J. C., García-Balderas, C. M. \& Lucio-Palacio, C. R. 2012. Cuatro nuevas adiciones para la araneofauna del estado de Guanajuato, México. Acta Zoológica Mexicana (n.s.), 28: 491-495.

Campuzano-Granados E. F. 2012. Araneofauna del suelo (Arachnida: Araneae) de Coatepec Harinas, Estado de México. Tesis de Licenciatura, Facultad de Ciencias, Universidad Autónoma del Estado de México, México, 136 pp.

Coddington, J. A. \& Levi, H. W. 1991. Systematics and evolution of spiders. Annual Review of Ecology and Systematics, 22: 565-592.

CONABIO. (2012). http://www.conabio.gob.mx/informacion/gis/. Consultado el 9 de agosto de 2013.

Correa-Ramírez, M. M., Jiménez, M. L. \& García-De León, F. J. 2010. Testing species boundaries in Pardosa sierra (Araneae: Lycosidae) using female morphology and COI mtDNA. Journal of Arachnology, 38: 538-554.

Coyle, F. A. 1988. A revision of the American Funnel-Web Mygalomorph spider genus Euagrus (Araneae: Dipluridae). Bulletin of American Museum of Natural History, 187: 203-292.

Cruz, D. A. 2014. Biodiversidad de arañas (Arachnida:Araneae) del Parque Estatal Sierra de Guadalupe. Tesis de Licenciatura, Escuela Nacional de Ciencias Biológicas, Instituto Politécnico Nacional, México, $177 \mathrm{pp}$.

Desales-Lara, M. A. 2009. Arañas (Arachnida:Araneae) de Malinalco, Estado de México. Tesis de Licenciatura, Facultad de Ciencias, Universidad Autónoma del Estado de México, México, 111 pp.

Desales-Lara, M. A. 2011. Diversidad de arañas (Arachnida:Araneae) en hábitats antropogénicos. Tesis de Maestría, Facultad de Ciencias, Universidad Autónoma del Estado de México, México, 56 pp.

Desales-Lara. M. A. 2012. Descripción de una nueva especie del género Filistatinella (Araneae: Filistatidae) de Toluca Estado de México. Revista Ibérica de Aracnología, 21: 51-55.

Desales-Lara, M. A., Durán-Barrón, C. G. \& Mulia-Solano, C. E. 2008. Nuevos registros de aranéidos y terídiidos (Araneae: Araneidae, Theridiidae) del Estado de México. Dugesiana, 15: 1-6.

Desales-Lara, M. A., Francke, O. \& Sánchez, P. 2013. Diversidad de Arañas (Arachnida: Araneae) en Hábitats Antropogénicos. Revista Mexicana de Biodiversidad, 84: 291-305.

Durán-Barrón, C. G. 2004. Diversidad de arañas (Arachnida, Araneae) asociadas a viviendas de la Ciudad de México. Tesis de Maestría, Instituto de Biología, Universidad Nacional Autónoma de México, México, 229 pp.

Durán-Barrón, C. G., Francke, O. F. \& Pérez-Ortiz, T. M. 2009. Diversidad de arañas (Arachnida: Araneae) asociadas a viviendas de la Ciudad de México (Área metropolitana). Revista Mexicana de Biodiversidad, 80: 55-69.

Estrada-Alvarez, J. C. \& Locht, A. 2011. Descripción de Bonnetina aviae sp. n. de México (Araneae: Theraphosidae: Theraphosinae). Boletín de la Sociedad Entomológica Aragonesa, 48: 151155. 
Francke B., O. F. 2011. La Aracnología en México: Pasado, Presente y Futuro (p. 43-50). Memorias y resúmenes del III Congreso Latinoamericano de Aracnología, Quindio, Colombia. 252 pp.

García-Villafuerte, M. A. 2009. La araneofauna (Araneae) reciente y fósil de Chiapas, México. Revista Mexicana de Biodiversidad, 80: 633- 646.

Gertsch, W. J. \& Ennik, F. 1983. The Spiders Genus Loxosceles in North America, Central America and the West Indies. Bulletin of American Museum of Natural History, 175: 264-360.

Guerrero-Fuentes, D. R. 2014. Diversidad de arañas del clado RTA (Arachnida: Araneae) de Tonatico, Estado de México. Tesis de Licenciatura, Facultad de Ciencias, Universidad Autónoma del Estado de México, México, 149 pp.

Guerrero-Fuentes, D. \& Desales-Lara, M. A. 2013. Categorización ecológica de las familias de arañas (Arachnida: Araneae) en tres localidades del municipio de Tonatico, Estado de México, pp. 166171. In: A. Equihua, E. Estrada, J. Acuña \& M. Chaires (Eds). Entomología Mexicana. Vol. 12. Sociedad Mexicana de Entomología, México.

Hoffmann, A. 1976. Relación bibliográfica preliminar de las arañas de México (Arachnida: Araneae). Publicaciones Especiales No. 3, Instituto de Biología, Universidad Nacional Autónoma de México, México, 117 pp.

Ibarra-Núñez, G., Maya-Morales, J., Chamé-Vázquez, D. 2011. Las arañas del bosque mesófilo de montaña de la Reserva de la Biosfera Volcán Tacaná, Chiapas, México. Revista Mexicana de Biodiversidad, 82: 1183-1193.

Jiménez, M-L. 1985a. Descripción de una nueva especie de la familia Thomisidae (Arachnida: Araneae) de México. Anales del Instituto de Biología. 56. Serie de Zoología, 1: 11-14.

Jiménez, M-L. 1985b. Nuevas descripciones de especies del género Pardosa grupo distincta (Araneae: Lycosidae). Folia Entomológica Mexicana, 70: 123-129.

Jiménez, M-L. 1986. Descripciones de arañas del género Pardosa grupo "Distincta" (Araneae: Lycosidae). Folia Entomológica Mexicana, 70: 123-129.

Jiménez, M-L. 1987. Dos nuevas arañas cangrejo (Araneae, Thomisidae) de México. Journal of Arachnology, 15: 395-399.

Jiménez, M-L., 1996. Araneae, pp: 83-101. In: Llorente Bousquets, J., García Aldrete, N. A. \& González Soriano, E. (Eds.). Biodiversidad, Taxonomía y Biogeografía de los artrópodos de México: Hacia una sintesis de su conocimiento. UNAM, CONABIO, México.

Jiménez-Jiménez, M. L. 1989. Las arañas Araneomorphae de San Francisco Oxtotilpan Estado de México. Tesis Doctoral, Facultad de Ciencias, Universidad Nacional Autónoma de México, México, $303 \mathrm{pp}$.

Jiménez, M-L. \& Morales, M. L. 1986. Descripción de una nueva especie del género Dictyna (Dictynidae: Araneae). Anales del Instituto de Biología, Serie Zoología, 56: 693-696.

Levi H. W. 2005. The orb-weaver genus Mangora of Mexico, Central America, and the West Indies (Araneae: Araneidae). Bulletin of the Museum of Comparative Zoology, 158: 139-182.

Lucio-Palacio, C. R. 2012. Nuevos registros de arañas errantes para el estado de Aguascalientes, México. Dugesiana, 19: 35-36.

Medina, F. J. 2002. Las arañas y su distribución temporal en un bosque de San Martín Cachihuapan, Municipio de Villa del Carbón, Estado de México. Tesis de Licenciatura, Universidad Nacional Autónoma de México, México, 129 pp.

Mendoza, J. I. 2014. Taxonomic revision of Hemirrhagus Simon, 1903 (Araneae: Theraphosidae, Theraphosinae), with description of five new species from Mexico. Zoological Journal of the Linnean Society, 170: 634-689.

Olguín, L. P. 2004. Catálogo de las arañas de la familia Lycosidae Sundevall, depositada en la Colección Nacional de Arácnidos (CNAN). Tesis de Licenciatura, Facultad de Ciencias, Universidad Nacional Autónoma de México, México, 87 pp. 
Platnick, N. I. 1977. New Species and records of the Anyphaena ceter group in México (Araneae: Anyphaenidae). Journal of Arachnology, 4: 207-210.

Platnick, N. I. 2014. The World Spider Catalog, version 14.5. American Museum of Natural History, online at http://research.amnh.org/iz/spiders/catalog. Doi: 10.5531/db.iz.0001.

Platnick, N. I. \& Shadab, M. U. 1977. A revision of the Spider Genera Herpyllus and Scotophaeus (Araneae: Gnaphosidae). Bulletin of American Museum of Natural History, 159: 1-44.

Platnick, N. I. \& Shadab, M. U. 1980. A revision of the Spider Genus Cesonia (Araneae: Gnaphosidae). Bulletin of American Museum of Natural History, 165: 337-385.

Platnick, N. I. \& Shadab, M. U. 1982. A revision of the American Spiders of the Genus Drasyllus (Araneae: Gnaphosidae). Bulletin of American Museum of Natural History, 173: 1-97.

Platnick, N. I. \& Ubick, D. 2007. A revision of the Spiders Genus Zorocrates Simon (Araneae, Zorocratidae). American Museum Novitates, 3579: 1-44.

Rheims, C. A., Brescovit, A. D. \& Durán-Barrón, C. G. 2006. Mexican species of the genus Scytodes Latreille (Araneae, Scytodidae). Revista Ibérica de Aracnología, 13: 93-119.

Tapia-Rodríguez, A. 2013. Diversidad de arañas (Arachnida: Araneae) de la Sierra de Nanchititla, Estado de México. Tesis de Licenciatura. Universidad Autónoma del Estado de México. 100 pp.

Valdez-Mondragón, A. 2010. Revisión taxonómica de Physocyclus Simon, 1893 (Araneae: Pholcidae), con la descripción de especies nuevas de México. Revista Ibérica de Aracnología, 18: 3-80. 